\title{
Predictors of Three-month Hospital Readmissions and Mortality in Patients with Cirrhosis of Liver
}

\author{
Anurag Lavekar ${ }^{1}$, Dhananjay Raje ${ }^{2}$, Aarsha Sadar $^{3}$, Tanuja Manohar ${ }^{4}$, Kavutharapu Sri Manjari ${ }^{5}$, Pradeep T Satyanarayana ${ }^{6}$
}

\begin{abstract}
Background: The rate of readmission to the hospital and mortality within 3 months is used as a quality measure for hospitalized patients with advanced liver disease; however, the topic has not been studied adequately under Indian context.

Materials and methods: This study was a longitudinal study conducted from March 2017 to March 2018. Patients admitted with liver cirrhosis at inpatient hepatology service in Tertiary Health Care Centre, Mysore, India, were included for the study. A total of 232 patients were studied and their demographic, clinical, biochemical parameters along with readmission status and outcomes within 3 months of observation were recorded. The effect of these factors on readmission and mortality was studied through multivariate logistic regression.

Results: The risk of readmission within 3 months was significantly associated with the presence of hydrothorax, hepatorenal syndrome (HRS), and portal vein thrombosis (PVT). Maddrey's discriminant function (DF), model for end-stage liver disease (MELD) score, and the Child-TurcottePugh (CTP) C grade also significantly increased the odds of readmission. The area under curve (AUC) for DF and MELD were 0.927 and 0.928 , respectively. Both DF and MELD significantly increased the odds of mortality.

Conclusion: The present study revealed that the parameters such as MELD and DF score and complications such as hydrothorax, HRS, and PVT are the most predictive indicators of cirrhosis complication to ascertain the rate of readmission and mortality within 3 months of patient discharge. Keywords: Cirrhosis, Liver disorders, Model for end-stage liver disease, Readmission.

Euroasian Journal of Hepato-Gastroenterology (2019): 10.5005/jp-journals-10018-1302
\end{abstract}

\section{INTRODUCTION}

The worldwide prevalence of cirrhosis of liver ranges from 4.5 to $9.5 \%$ in the general population. ${ }^{1-3}$ This prevalence may be an underrepresentation of the actual disease burden as up to one third of the cases remain asymptomatic till the disease progresses to an advanced stage. It is also a leading cause of mortality in the developed as well as developing countries. Mortality due to cirrhosis of liver is expected to increase, and it is expected to be the 12th leading cause of death all over the world by $2020 .{ }^{4}$ Cirrhosis of liver also contributes significantly to the morbidity due to complications, increased burden on healthcare system, frequent readmissions and economic, psychological and social burden on patients as well as family members. ${ }^{5,6}$ Increased frequency of admissions not only increases the financial burden on patients but also makes them apprehensive and subvert their credence of the treating physician. In some of the chronic disease states (e.g., congestive cardiac failure), early readmissions are well recognized, partially preventable because of successful interventions..$^{7-9}$ However, with cirrhosis of liver, characteristics of readmission and mortality are still evolving. It becomes imperative to study the profile of admitted cirrhosis cases to understand the predictors of readmission and mortality. Several researchers across the globe have attempted to study such predictors. ${ }^{10-15}$ However, data in this regard are lacking for India. Our study aims at studying the predictors of readmission rate and mortality within 3 months of hospital discharge. For a chronic disease such as cirrhosis, it has always been challenging for physicians to elaborate reliable tools for predicting the outcome. Most of the tools developed are for prediction of short-term mortality and morbidity assessments and predictions. For decades, the available methods included tools like CTP score that relied on a limited number of variables, which, based on clinical experience, are
${ }^{1,3}$ Department of Gastroenterology and Hepatology, JSS Medical College and Hospital, Mysore, Karnataka, India

${ }^{2}$ Department of Biostatistics, Data Analysis Group, MDS Bio Analytics Private Limited, Nagpur, Maharashtra, India

${ }^{4}$ Department of General Medicine, NKP Salve Institute of Medical Sciences and Lata Mangeshkar Hospital, Nagpur, Maharashtra, India

${ }^{5}$ Department of Genetics and Biotechnology, University College for Women, Koti, Hyderabad, Telangana, India

${ }^{6}$ Department of Community Medicine, Sri Devaraj Urs Medical College, Sri Devaraj Urs Academy of Higher Education and Research, Kolar, Karnataka, India

Address reprint requests to: Pradeep T Satyanarayana, Department of Community Medicine, Sri Devaraj Urs Medical College, Sri Devaraj Urs Academy of Higher Education and Research, Kolar, Karnataka, India, Phone: +91 7892950465, e-mail: dr.pradeep.ts@gmail.com

How to cite this article: Lavekar A, Raje D, Sadar A, et al. Predictors of Three-month Hospital Readmissions and Mortality in Patients with Cirrhosis of Liver. Euroasian J Hepato-Gastroenterol 2019;9(2):71-77.

Source of support: Nil

Conflict of interest: None

routinely used to determine the prognosis of patients with limited success in short-term setting. But recently more emphasis is given to variables that are shown to be significantly and independently correlated to the outcome by multivariate analysis. One of the most common examples is MELD score, which is now preferred over CTP score at many centers. ${ }^{16}$ The current evidence suggests that approximately half of the patients get readmitted due to complications related to liver cirrhosis within 3 months of hospital

() The Author(s). 2019 Open Access This article is distributed under the terms of the Creative Commons Attribution 4.0 International License (https://creativecommons. org/licenses/by-nc/4.0/), which permits unrestricted use, distribution, and non-commercial reproduction in any medium, provided you give appropriate credit to the original author(s) and the source, provide a link to the Creative Commons license, and indicate if changes were made. The Creative Commons Public Domain Dedication waiver (http://creativecommons.org/publicdomain/zero/1.0/) applies to the data made available in this article, unless otherwise stated. 
discharge. Many readmissions are related to potentially modifiable factors. Therefore, efforts at discharge must focus on this high-risk group of patients to reduce the readmission rate. ${ }^{17}$

\section{Materials and Methods}

This study was a longitudinal study conducted from March 2017 to March 2018. Institutional Ethical Committee approval was taken. Patients admitted with liver cirrhosis at a tertiary healthcare center were included in this study after obtaining informed consent from patient or patient's relative if the patient is not in a condition to give consent. Patients who were admitted for an elective indication, with a uncertain diagnosis of cirrhosis of liver, and unwilling to participate in the study were excluded.

After recording the preliminary data such as name, age, gender, and etiology of liver cirrhosis, biochemical parameters such as complete blood count, liver function test, renal function tests, prothrombin time (PT), international normalized ratio (INR), etc., were also recorded. Complications due to cirrhosis and portal hypertension such as presence gastrointestinal (GI) bleed, hepatic encephalopathy (HE), spontaneous bacterial peritonitis (SBP), HRS, and hepatic hydrothorax were also observed. Moreover, presence of infections (pneumonia, urinary tract infections, SBP, cellulitis, soft tissue infections, etc.) and associated comorbidities was recorded. Maddrey's DF, MELD score and CTP score were calculated using the standard formula. Included patients were followed up for 3 months through phone calls and medical records. Readmission and deaths were recorded. Demographic, clinical, and biochemical profiles of patients were summarized according to two independent outcome events, viz. (a) readmission within 3 months and (b) mortality.

The statistical significance of difference was obtained using Pearson's chi-square test for categorical parameters, while independent samples' $t$ test was used for continuous parameters. The parameters showing significant difference between the groups in univariate analysis were considered for multivariate logistic regression model. Hosmer-Lemeshow test was referred to decide the goodness-of-fit of multivariate model. The adjusted odds ratio (OR) obtained through multivariate model were referred to determine the risk of events associated with different parameters. Receiveroperating characteristic (ROC) analysis was performed to determine the prognostic accuracy of continuous parameters having significant effect on the outcome variables. The cutoff values of parameters were obtained and their sensitivity and specificity were determined. All the analyses were performed using SPSS version 20.0 (IBM Corp. Armonk, USA), and the statistical significance was tested at $5 \%$ level.

\section{Results}

The mean age of patients in the study was $49.6 \pm 12.8$ years. Among 232 patients, 187 (80.6\%) were males and 45 (19.4\%) were females. As regards readmission, 89 (38.4\%) patients were readmitted within 3 months. The demographic profile of patients in admitted and nonadmitted groups was insignificantly different. Alcohol was the prominent etiology in both the groups. The proportion of patients with GI bleed and their distribution according to the grade of varices was insignificantly different in two groups. The proportion of cases with $\mathrm{HE}$ in readmission group (48.3\%) was significantly higher than the group not requiring readmission. Moreover, the proportion of cases with hydrothorax, HRS, PVT, and hepatocellular carcinoma (HCC) were significantly higher in readmitted patients, which was statistically significant (Table $1 \mathrm{~A}$ ).
As regards mortality outcome, there were 34 (14.6\%) deaths in the study sample. The demographic profile of mortality cases was insignificantly different than the nonmortality group. Proportion of cases with GI bleed was significantly lower in the mortality group (17.65\%) as compared to other group (36.87\%), which was statistically significant. The distribution of patients per the grade of varices was insignificantly different in the two groups. Other parameters such as HE, hydrothorax, HRS, PVT, and HCC showed significantly higher proportion in mortality group as compared to other group with $p<0.05$ (Table 1B).

Table 2 provides the comparison of various biochemical parameters between two groups for each outcome, i.e., readmission and death within 3 months of hospital discharge. The mean values of total blood cell counts and total and direct bilirubin were significantly higher in the readmitted group as indicated by the corresponding $p<0.05$. The mean platelet count was significantly lower in the readmission group ( $p=0.003)$. The ratio of AST and ALT was significantly higher in the readmission group as compared to the other group $(p<0.0001)$. Other parameters such as PT, INR, urea, creatinine, $\mathrm{Na}, \mathrm{DF}$, and MELD also had significantly higher mean levels in the readmission group compared to the other group ( $p<$ 0.05 ). The CTP classification in two groups suggested significantly different distribution of patients in two groups $(p<0.0001)$. The proportion of readmitted cases with CTP category C (70.8\%) was significantly higher than that of the other group.

Further, all the parameters were also studied using mortality as an outcome. The parameters showing significant difference between mortality and nonmortality groups were almost the same as observed for readmission within 3 months, except AST/ ALT, albumin, and Na. Additionally, parameters Gl bleed and potassium (K) showed significant difference between the two categories (Table 3).

The risk of outcomes associated with demographic, clinical, and biochemical parameters was also determined through multivariate logistic regression analysis. The parameters showing significant difference between comparison groups were included in the multivariate model. Parameters PT and INR showed high collinearity with $\mathrm{DF}$, and hence both were ignored from the model. Also, total and direct bilirubin showed high correlation, and hence only total bilirubin was retained in the model. The risk of readmission within 3 months was significantly associated with the presence of hydrothorax and HRS with ORs of 18.386 and 24.573, respectively, compared to those without such syndrome. The PVT also showed significantly increased risk of readmission with ORs 5.3. Further, unit increase in DF and MELD score significantly increased the risk of readmission by 1.05 and 1.2 times, respectively. The CTP C grade showed increased risk of readmission with OR of 17.2 as compared to grade I. The remaining parameters showed insignificant effect on the outcome as revealed through multivariate model.

For continuous parameters DF and MELD, which had significant effect on hospital readmission, the ROC analysis was performed to determine their respective cutoffs and their prognostic accuracy. Figure 1 provides ROC plots for the two parameters. The AUC for DF and MELD were 0.927 (95\% confidence interval (CI): 0.89-0.964; $p$ $<0.0001)$ and 0.928 (95\% Cl: 0.895-0.960; $p<0.0001)$, respectively, which were almost similar. For DF, a threshold score of $49.96 \mathrm{had}$ a sensitivity of 0.81 and specificity of 0.91 , which was optimum according to Youden's index. For MELD, a threshold score of 17.5 had a sensitivity of 0.92 and specificity of 0.79 . 
Table 1A: Descriptive statistics for various parameters according to readmission status

\begin{tabular}{|c|c|c|c|c|}
\hline \multirow[b]{2}{*}{ Parameters } & & \multicolumn{2}{|c|}{$\begin{array}{c}\text { Readmission within } 3 \\
\text { months }\end{array}$} & \multirow[b]{2}{*}{$p$ value } \\
\hline & & No $(n=143)$ & Yes $(n=89)$ & \\
\hline \multicolumn{2}{|c|}{ Age in years (mean $\pm S D$ ) } & $49.86 \pm 13.79$ & $49.31 \pm 11.10$ & 0.751 \\
\hline \multirow[t]{2}{*}{ Sex [no. (\%)] } & Female & $33(23.1)$ & $12(13.5)$ & 0.104 \\
\hline & Male & $110(76.9)$ & $77(86.5)$ & \\
\hline \multirow{8}{*}{$\begin{array}{l}\text { Etiology } \\
\text { [no. (\%)] }\end{array}$} & Alcohol & $89(62.2)$ & 69 (77.5) & 0.131 \\
\hline & EHPVO & $2(1.4)$ & $0(0)$ & \\
\hline & HBV & $15(10.5)$ & $4(4.5)$ & \\
\hline & $\mathrm{HBV}+\mathrm{HCV}$ & $1(0.7)$ & $0(0)$ & \\
\hline & $\mathrm{HCV}$ & $7(4.9)$ & $4(4.5)$ & \\
\hline & $\mathrm{HIV}+\mathrm{HBV}$ & $0(0)$ & $1(1.1)$ & \\
\hline & NAFLD & $24(16.8)$ & $7(7.8)$ & \\
\hline & NCPF & $5(3.5)$ & $4(4.5)$ & \\
\hline \multirow{2}{*}{$\begin{array}{l}\text { Gl bleed } \\
\text { [no. (\%)] }\end{array}$} & No & $90(62.9)$ & $63(70.8)$ & 0.278 \\
\hline & Yes & $53(37.1)$ & $26(29.2)$ & \\
\hline \multirow{3}{*}{$\begin{array}{l}\text { Grade of } \\
\text { varices } \\
\text { [no. (\%)] }\end{array}$} & I & $84(58.7)$ & $43(48.3)$ & 0.296 \\
\hline & II & $35(24.5)$ & $28(31.5)$ & \\
\hline & III & $24(16.8)$ & $18(20.2)$ & \\
\hline \multirow[t]{2}{*}{$\mathrm{HE}[$ no. (\%)] } & No & $121(84.6)$ & $46(51.7)$ & $<0.0001$ \\
\hline & Yes & $22(15.4)$ & $43(48.3)$ & \\
\hline \multirow{2}{*}{$\begin{array}{l}\text { Hydrothorax } \\
\text { [no. (\%)] }\end{array}$} & No & $141(98.6)$ & $71(79.8)$ & $<0.0001$ \\
\hline & Yes & $2(1.40)$ & $18(20.2)$ & \\
\hline \multirow[t]{2}{*}{ HRS [no. (\%)] } & No & $133(93.0)$ & $54(60.7)$ & $<0.0001$ \\
\hline & Yes & $10(7.0)$ & 35 (39.3) & \\
\hline \multirow[t]{2}{*}{ PVT [no. (\%] } & No & $123(86.0)$ & 53 (59.6) & $<0.0001$ \\
\hline & Yes & $20(14.0)$ & $36(40.4)$ & \\
\hline \multirow[t]{2}{*}{ HCC [no. (\%)] } & No & 139 (97.2) & 76 (85.4) & 0.002 \\
\hline & Yes & $4(2.8)$ & $13(14.6)$ & \\
\hline \multicolumn{5}{|c|}{$\begin{array}{l}\text { SD, standard deviation; HBV, hepatitis B virus; } \mathrm{HCV} \text {, hepatitis } \mathrm{C} \text { virus; } \mathrm{Gl} \text {, } \\
\text { gastrointestinal; } \mathrm{HE} \text {, hepatic encephalopathy; } \mathrm{HRS} \text {, hepatorenal syndrome; } \\
\text { PVT, portal vein thrombosis; } \mathrm{HCC} \text {, hepatocellular carcinoma; EHPVO, extra- } \\
\text { hepatic portal vein obstruction; NAFLD, nonalcoholic fatty liver disease; } \\
\text { NCPF, noncirrhotic portal fibrosis } \\
\text { p values in boldface indicate statistical significance }\end{array}$} \\
\hline \multicolumn{5}{|c|}{$\begin{array}{l}\text { Of the } 89 \text { cases of readmission within } 3 \text { months, } 34 \text { ( } 38.2 \% \text { ) had } \\
\text { ortality during hospital stay. The risk of mortality associated with } \\
\text { e presence of HRS was } 8.652 \text { ( } 95 \% \text { Cl: } 1.946-38.463 ; p=0.005 \text { ) times } \\
\text { gher as compared to those without HRS. Further, a unit increase in } \\
\text { and MELD significantly increased the risk of mortality } 1.025 \text { ( } 95 \% \\
1.005-1.044 ; p=0.013 \text { ) and } 1.115 \text { ( } 95 \% \text { Cl: } 1.000-1.246 ; p=0.048) \\
\text { nes. Remaining parameters had statistically insignificant effect } \\
\text { mortality. The ROC analysis was also performed on these two } \\
\text { rameters with mortality as an event. The AUC for DF and MELD was } \\
384 \text { ( } 95 \% \text { Cl: } 0.805-0.962 ; p<0.0001) \text { and } 0.902 \text { ( } 95 \% \text { Cl: } 0.841-0.963 \text {; } \\
\text { < } 0.0001) \text {. The threshold for DF was } 64.10 \text { with a sensitivity of } 0.88 \\
\text { d specificity of } 0.85 ; \text { while for MELD it was } 26.5 \text { with a sensitivity } \\
0.74 \text { and specificity of } 0.92 \text {. The criteria for DF and MELD can be } \\
\text { ed in conjunction to decide the prognosis of outcomes (Fig. } 2 \text { ). }\end{array}$} \\
\hline
\end{tabular}

\section{Discussion}

In the current study, cirrhotic patients hospitalized for nonelective indications were consented and followed for 3 months post
Table 1B: Descriptive statistics for various parameters according to mortality

\begin{tabular}{|c|c|c|c|c|}
\hline \multirow{2}{*}{ Parameters } & & \multicolumn{2}{|c|}{ Mortality } & \multirow[b]{2}{*}{$p$ value } \\
\hline & & No $(n=198)$ & $\operatorname{Yes}(n=34)$ & \\
\hline \multicolumn{2}{|c|}{ Age in years (mean $\pm S D)$} & $50 \pm 13$ & $49 \pm 10$ & 0.684 \\
\hline \multirow[t]{2}{*}{ Sex [no. (\%)] } & Female & $40(20.2)$ & $5(14.71)$ & 0.454 \\
\hline & Male & $158(79.8)$ & 29 (85.29) & \\
\hline \multirow{8}{*}{$\begin{array}{l}\text { Etiology } \\
\text { [no. (\%)] }\end{array}$} & Alcohol & $129(65.1)$ & $29(85.3)$ & 0.508 \\
\hline & EHPVO & $2(1.0)$ & $0(0)$ & \\
\hline & HBV & $17(85.8)$ & $2(5.9)$ & \\
\hline & $\mathrm{HBV}+\mathrm{HCV}$ & $1(0.5)$ & $0(0)$ & \\
\hline & $\mathrm{HCV}$ & $10(5.1)$ & $1(2.9)$ & \\
\hline & $\mathrm{HIV}+\mathrm{HBV}$ & $1(0.5)$ & $0(0)$ & \\
\hline & NAFLD & $30(15.1)$ & $1(2.9)$ & \\
\hline & NCPF & $8(4.0)$ & $1(2.9)$ & \\
\hline \multirow{2}{*}{$\begin{array}{l}\text { Gl bleed } \\
{[\text { no. (\%)] }}\end{array}$} & No & $125(63.13)$ & $28(82.35)$ & 0.029 \\
\hline & Yes & $73(36.87)$ & $6(17.65)$ & \\
\hline \multirow{3}{*}{$\begin{array}{l}\text { Grade of } \\
\text { varices [no. (\%)] }\end{array}$} & I & $108(54.5)$ & $19(55.8)$ & 0.844 \\
\hline & ॥ & $53(26.7)$ & $10(29.4)$ & \\
\hline & III & 37 (18.7) & $5(14.7)$ & \\
\hline \multirow[t]{2}{*}{ HE [no. (\%)] } & No & $153(77.27)$ & $14(41.18)$ & $<0.0001$ \\
\hline & Yes & $45(22.73)$ & $20(58.82)$ & \\
\hline \multirow{2}{*}{$\begin{array}{l}\text { Hydrothorax } \\
\text { [no. (\%)] }\end{array}$} & No & $186(93.94)$ & $26(76.47)$ & 0.001 \\
\hline & Yes & $12(6.06)$ & $8(23.53)$ & \\
\hline \multirow[t]{2}{*}{ HRS [no. (\%)] } & No & $173(87.37)$ & $14(41.18)$ & $<0.0001$ \\
\hline & Yes & $25(12.63)$ & $20(58.82)$ & \\
\hline \multirow[t]{2}{*}{ PVT [no. (\%] } & No & 156 (78.79) & $20(58.82)$ & 0.012 \\
\hline & Yes & $42(21.2)$ & $14(41.18)$ & \\
\hline \multirow[t]{2}{*}{ HCC [no. (\%)] } & No & $192(96.97)$ & $23(67.65)$ & $<0.0001$ \\
\hline & Yes & $6(3.03)$ & $11(32.35)$ & \\
\hline
\end{tabular}

SD, standard deviation; HBV, hepatitis B virus; HCV, hepatitis C virus; Gl, gastrointestinal; $\mathrm{HE}$, hepatic encephalopathy; $\mathrm{HRS}$, hepatorenal syndrome; PVT, portal vein thrombosis; HCC, hepatocellular carcinoma; EHPVO, extrahepatic portal vein obstruction; NAFLD, nonalcoholic fatty liver disease; NCPF, noncirrhotic portal fibrosis

$p$ values in boldface indicate statistical significance

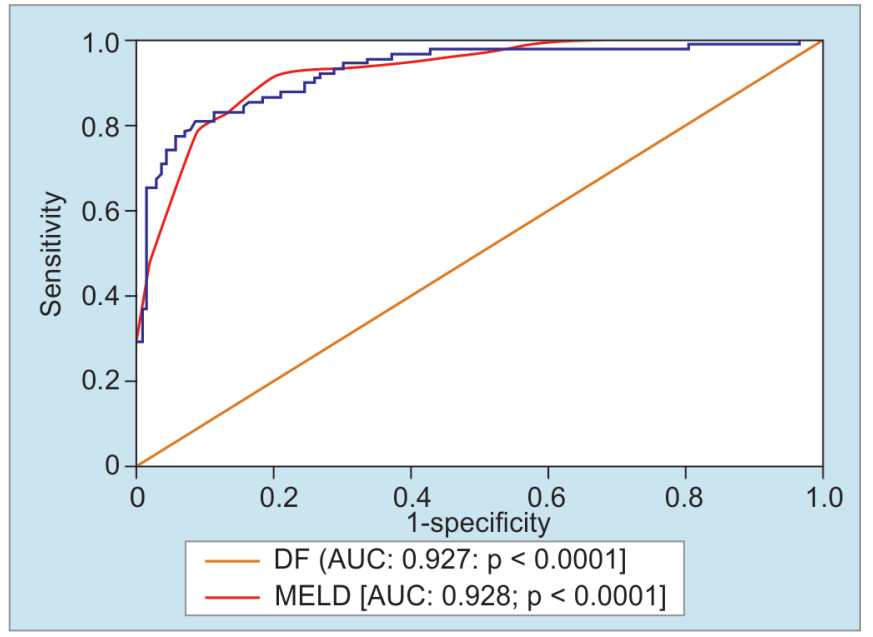

Fig. 1: Receiver-operating characteristic plots for readmission within 3 months for parameters Maddrey's discriminant function and model for end-stage liver disease 
Table 2A: Descriptive statistics for various parameters according to the readmission status

\begin{tabular}{|c|c|c|c|c|}
\hline & \multirow[b]{2}{*}{ Parameters } & \multicolumn{2}{|c|}{ Readmission within 3 months } & \multirow[b]{2}{*}{ pvalue } \\
\hline & & No $(n=143)$ & Yes $(n=89)$ & \\
\hline 1 & $\mathrm{Hb}(\mathrm{M} \pm \mathrm{SD})$ & $9.33 \pm 2.63$ & $9.14 \pm 2.72$ & 0.602 \\
\hline 2 & $\mathrm{TLC}(\mathrm{M} \pm \mathrm{SD})$ & $8472.24 \pm 4297.7$ & $12314.27 \pm 6668.5$ & $<0.0001$ \\
\hline 3 & $N(M \pm S D)$ & $73.51 \pm 11.3$ & $78.33 \pm 11.33$ & 0.002 \\
\hline 4 & $\begin{array}{l}\text { Platelets } \\
(\mathrm{M} \pm \mathrm{SD})\end{array}$ & $1.51 \pm 0.99$ & $1.14 \pm 0.81$ & 0.003 \\
\hline 5 & $\begin{array}{l}\text { T bilirubin } \\
(M \pm S D)\end{array}$ & $3.29 \pm 4.34$ & $10.53 \pm 8.32$ & $<0.0001$ \\
\hline 6 & $\begin{array}{l}\text { D bilirubin } \\
(M \pm S D)\end{array}$ & $1.93 \pm 3.17$ & $5.94 \pm 5.39$ & $<0.0001$ \\
\hline 7 & $\mathrm{AST}(\mathrm{M} \pm \mathrm{SD})$ & $135.07 \pm 648.36$ & $162.37 \pm 337.09$ & 0.714 \\
\hline 8 & $\operatorname{ALT}(M \pm S D)$ & $78.55 \pm 265.79$ & $102.97 \pm 261.4$ & 0.494 \\
\hline 9 & $\begin{array}{l}\text { AST:ALT R } \\
(M \pm S D)\end{array}$ & $1.59 \pm 0.63$ & $2.05 \pm 0.85$ & $<0.0001$ \\
\hline 10 & $\operatorname{ALP}(M \pm S D)$ & $358.38 \pm 336.88$ & $329.17 \pm 172$ & 0.449 \\
\hline 11 & $\begin{array}{l}\text { Protein } \\
(M \pm S D)\end{array}$ & $6.11 \pm 0.85$ & $6.21 \pm 0.8$ & 0.364 \\
\hline 12 & $\begin{array}{l}\text { Albumin } \\
(M \pm S D)\end{array}$ & $3.19 \pm 0.57$ & $2.95 \pm 0.57$ & 0.003 \\
\hline 13 & $\mathrm{PT}(\mathrm{M} \pm \mathrm{SD})$ & $19.31 \pm 3.66$ & $31.78 \pm 12.82$ & $<0.0001$ \\
\hline 14 & $\mathrm{INR}(\mathrm{M} \pm \mathrm{SD})$ & $1.41 \pm 0.32$ & $2.54 \pm 1.21$ & $<0.0001$ \\
\hline 15 & $\begin{array}{l}\text { Urea } \\
(M \pm S D)\end{array}$ & $38.99 \pm 23.04$ & $50.84 \pm 37.83$ & 0.003 \\
\hline 16 & $\begin{array}{l}\text { Creatinine } \\
(M \pm S D)\end{array}$ & $1.05 \pm 0.49$ & $1.64 \pm 1.33$ & $<0.0001$ \\
\hline 17 & $\mathrm{Na}(\mathrm{M} \pm \mathrm{SD})$ & $135.61 \pm 6.98$ & $133.29 \pm 7.47$ & 0.018 \\
\hline 18 & $K(M \pm S D)$ & $4.16 \pm 0.68$ & $4.16 \pm 1.02$ & 0.963 \\
\hline 19 & $\mathrm{DF}(\mathrm{M} \pm \mathrm{SD})$ & $27.76 \pm 16.91$ & $91.71 \pm 60.48$ & $<0.0001$ \\
\hline 20 & $\begin{array}{l}\text { MELD } \\
(M \pm S D)\end{array}$ & $14.36 \pm 4.59$ & $26.51 \pm 8.0$ & $<0.0001$ \\
\hline 21 & $\begin{array}{l}\text { CTP } \\
\text { [no. (\%)] A }\end{array}$ & $63(44.1)$ & $4(4.5)$ & $<0.0001$ \\
\hline & B & $67(46.8)$ & $22(24.7)$ & \\
\hline & $\mathrm{C}$ & $13(9.1)$ & $63(70.8)$ & \\
\hline
\end{tabular}

$M$, mean; SD, standard deviation; $H B$, hemoglobin; INR, international normalized ratio; PT, prothrombin time; DF, discriminant function; MELD, model for end-stage liver disease; CTP, Child-Turcotte-Pugh; TLC, total leucocyte count; N, neutrophil; AST, aspartate transaminase; ALT, alanine transaminase; ALP, alkaline phosphatase; AST:ALT R, AST to ALT ratio $p$ values in boldface indicate statistical significance

discharge. Hospital readmission and short-term mortality in 3 months were studied. This study found that early rehospitalizations (38.36\%) and mortality (14.65\%) within 3 months of hospital discharge among patients with cirrhosis are common. The data from published literature from India reported 3 months hospital readmission at $42.30 \%$, while no data are available on mortality for general cirrhosis patients. ${ }^{17}$ Indian patients with severe alcoholic hepatitis showed a mortality rate of $44 \% .{ }^{18}$ By comparison, the rates of readmission at 3 months are generally higher than that in the Western population (30-35\%) $)^{13,14}$ and the mortality rate was found to be similar (16-18\%). . $^{19,20}$

The mean age ( 49.65 years) of study population was relatively younger by decade than the reported age-group of other Indian studies, which included all patients admitted in hospitals. ${ }^{12}$ But
Table 2B: Descriptive statistics for various parameters according to the mortality

\begin{tabular}{|c|c|c|c|c|}
\hline & \multirow[b]{2}{*}{ Parameters } & \multicolumn{2}{|c|}{ Mortality } & \multirow[b]{2}{*}{$p$ value } \\
\hline & & No $(n=198)$ & Yes $(n=34)$ & \\
\hline 1 & $\mathrm{Hb}(\mathrm{M} \pm \mathrm{SD})$ & $9.34 \pm 2.62$ & $8.79 \pm 2.88$ & 0.261 \\
\hline 2 & $\mathrm{TLC}(\mathrm{M} \pm \mathrm{SD})$ & $\begin{array}{l}9408.54 \pm \\
5259.56\end{array}$ & $\begin{array}{l}13076.76 \pm \\
6755.04\end{array}$ & $<0.0001$ \\
\hline 3 & $N(M \pm S D)$ & $74.38 \pm 11.44$ & $81.09 \pm 10.43$ & 0.002 \\
\hline 4 & $\begin{array}{l}\text { Platelets } \\
(\mathrm{M} \pm \mathrm{SD})\end{array}$ & $1.44 \pm 0.98$ & $0.95 \pm 0.43$ & 0.005 \\
\hline 5 & $\begin{array}{l}\text { T bilirubin } \\
(M \pm S D)\end{array}$ & $5.01 \pm 6.03$ & $12.22 \pm 9.48$ & $<0.0001$ \\
\hline 6 & $\begin{array}{l}\text { D bilirubin } \\
(M \pm S D)\end{array}$ & $3 \pm 4.33$ & $6.19 \pm 5.15$ & $<0.0001$ \\
\hline 7 & $\mathrm{AST}(\mathrm{M} \pm \mathrm{SD})$ & $129.88 \pm 553.41$ & $236.76 \pm 524.18$ & 0.296 \\
\hline 8 & $A L T(M \pm S D)$ & $74.75 \pm 228.22$ & $164.62 \pm 411.28$ & 0.066 \\
\hline 9 & $\begin{array}{l}\text { AST:ALT R } \\
(M \pm S D)\end{array}$ & $1.73 \pm 0.75$ & $1.96 \pm 0.92$ & 0.125 \\
\hline 10 & $\mathrm{ALP}(\mathrm{M} \pm \mathrm{SD})$ & $349.55 \pm 298.05$ & $333.32 \pm 195.27$ & 0.76 \\
\hline 11 & Protein $(M \pm S D)$ & $6.16 \pm 0.81$ & $6.04 \pm 0.96$ & 0.407 \\
\hline 12 & $\begin{array}{l}\text { Albumin } \\
(\mathrm{M} \pm \mathrm{SD})\end{array}$ & $3.13 \pm 0.57$ & $2.93 \pm 0.64$ & 0.062 \\
\hline 13 & $\mathrm{PT}(\mathrm{M} \pm \mathrm{SD})$ & $21.61 \pm 6.05$ & $38.56 \pm 16.84$ & $<0.0001$ \\
\hline 14 & $\mathrm{INR}(\mathrm{M} \pm \mathrm{SD})$ & $1.63 \pm 0.65$ & $3.09 \pm 1.43$ & $<0.0001$ \\
\hline 15 & Urea $(M \pm S D)$ & $40.57 \pm 25.52$ & $60.82 \pm 45.68$ & $<0.0001$ \\
\hline 16 & $\begin{array}{l}\text { Creatinine } \\
(M \pm S D)\end{array}$ & $1.15 \pm 0.72$ & $2.04 \pm 1.6$ & $<0.0001$ \\
\hline 17 & $\mathrm{Na}(\mathrm{M} \pm \mathrm{SD})$ & $134.91 \pm 7.22$ & $133.59 \pm 7.41$ & 0.325 \\
\hline 18 & $K(M \pm S D)$ & $4.11 \pm 0.72$ & $4.42 \pm 1.25$ & 0.047 \\
\hline 19 & $\mathrm{DF}(\mathrm{M} \pm \mathrm{SD})$ & $39.91 \pm 29.8$ & $124.44 \pm 78.74$ & $<0.0001$ \\
\hline 20 & $\operatorname{MELD}(M \pm S D)$ & $16.96 \pm 6.52$ & $31 \pm 8.93$ & $<0.0001$ \\
\hline \multirow[t]{3}{*}{21} & CTP [no. (\%)] A & $65(32.8)$ & $2(5.9)$ & $<0.0001$ \\
\hline & B & $84(42.4)$ & $5(14.7)$ & \\
\hline & C & $49(24.8)$ & $27(79.4)$ & \\
\hline
\end{tabular}

$M$, mean; SD, standard deviation; $H B$, hemoglobin; INR, international normalized ratio; PT, prothrombin time; DF, discriminant function; MELD, model for end-stage liver disease; CTP, Child-Turcotte-Pugh; TLC, total leucocyte count; N, neutrophil; AST, aspartate transaminase; ALT, alanine transaminase; ALP, alkaline phosphatase; AST:ALT R, AST to ALT ratio $p$ values in boldface indicate statistical significance

other recent studies have reported almost similar age-group with a range of 45-51 years. ${ }^{21,22}$ In the present study, age did not seem to have contributed to mortality or hospital readmission. Although mortality of $14.65 \%$ within 3 months was on high, it was supported by the fact that younger patients had more advanced disease at the time of hospital admission, as also indicated by the MELD score. ${ }^{23}$ Even though it was known that cirrhosis in older patients aged more than 65 years led to worse prognosis, with mortality seen in $>20 \%$ of admitted patients, ${ }^{23,24}$ and the trend seems to be not favoring just the older. We are unable to explain this surprising finding but speculate that either the biology of cirrhosis in young patients is different from that of the older patients or young patients generally come to hospital admission later in the course of their disease.

Similar nonsignificance was observed with gender. Published clinical study reports suggest that females require less intensive care management and have better outcome in comparison with males; the finding is not applicable for chronic and critical diseases 
Table 3A: Risk of readmission associated with different factors

\begin{tabular}{|c|c|c|c|c|c|}
\hline & & Readn & ssion wit & 3 months & \\
\hline & & & & for $O R$ & \\
\hline & Parameters & $O R^{*}$ & Lower & Upper & $p$ value \\
\hline 1 & Age & 1.036 & 0.982 & 1.094 & 0.1 \\
\hline 2 & Gender male & 2.769 & 0.582 & 13.177 & 0.2 \\
\hline 3 & Gl bleed yes & - & - & - & - \\
\hline 4 & HE yes & 0.948 & 0.255 & 3.527 & 0.9 \\
\hline 5 & Hydrothorax yes & 18.386 & 1.666 & 202.9 & 0.01 \\
\hline 6 & HRS yes & 24.573 & 3.635 & 166.1 & 0.001 \\
\hline 7 & PVT yes & 5.306 & 1.299 & 21.670 & 0.02 \\
\hline 8 & HCC yes & 1.763 & 0.068 & 45.545 & 0.7 \\
\hline 9 & $\mathrm{~N}$ & 0.988 & 0.941 & 1.037 & 0.6 \\
\hline 10 & Platelets & 0.970 & 0.466 & 2.019 & 0.9 \\
\hline 11 & Total bilirubin & 0.990 & 0.892 & 1.100 & 0.8 \\
\hline 12 & AST/ALT & 0.815 & 0.306 & 2.170 & 0.6 \\
\hline 13 & Albumin & 1.664 & 0.594 & 4.663 & 0.3 \\
\hline 14 & Urea & 0.996 & 0.964 & 1.029 & 0.8 \\
\hline 15 & Creatinine & 0.856 & 0.240 & 3.057 & 0.8 \\
\hline 16 & $\mathrm{Na}$ & 0.998 & 0.931 & 1.071 & 0.9 \\
\hline 17 & $\mathrm{~K}$ & - & - & - & - \\
\hline 18 & DF & 1.053 & 1.011 & 1.096 & 0.01 \\
\hline 19 & MELD & 1.238 & 1.028 & 1.492 & 0.02 \\
\hline 20 & CTP (B) & 5.101 & 0.400 & 65.050 & 0.2 \\
\hline 21 & CTP (C) & 17.276 & 1.295 & 230.51 & 0.031 \\
\hline
\end{tabular}

$\mathrm{OR}$, odds ratio; $\mathrm{Cl}$, confidence interval; $\mathrm{HBV}$, hepatitis $\mathrm{B}$ virus; $\mathrm{HCV}$, hepatitis C virus; $\mathrm{Gl}$, gastrointestinal; $\mathrm{HE}$, hepatic encephalopathy; $\mathrm{HRS}$, hepatorenal syndrome; PVT, portal vein thrombosis; HCC, hepatocellular carcinoma; INR, international normalized ratio; PT, prothrombin time; DF, discriminant function; MELD, model for end-stage liver disease; CTP, Child-TurcottePugh; AST, aspartate transaminase; ALT, alanine transaminase

$p$ values in boldface indicate statistical significance; ${ }^{*}$ obtained using multivariate logistic regression

such as cirrhosis. ${ }^{25}$ Female gender itself cannot be considered a protective factor; and in cirrhosis, the readmission and mortality rate were found similar to that of male patients.

Etiologies of cirrhosis did not seem to predict short-term re-admission or mortality of patients. Being an irreversible condition, with no known treatment for it, cirrhosis poses biggest challenge. Current study did not find predictive value of etiologies of cirrhosis for short-term mortality or re-admission. It is established that preventive measures such as hepatitis $B$ vaccination and reduced consumption of alcohol are beneficial. However, the measures are not applicable when cirrhosis has already set in. ${ }^{26}$

The liver function test parameters are routinely used for monitoring of patients, but their predictive values are best estimated for long-term patient outcomes. The elevated values have more significance in the absence of apparent liver diseases. ${ }^{27}$ Current study results did not find any deviation in the results from the established understanding.

Among complications of cirrhosis, HE has been considered an important predictor of short-term mortality and morbidity. It is an integral component of Child-Pugh score. Extensive data are published on its significance and management, because of the reversible nature of condition, leading to $\mathrm{HE}$ being the main focus of treatment for cirrhosis patients. ${ }^{16}$ Precipitating factors and
Table 3B: Risk of death associated with different factors

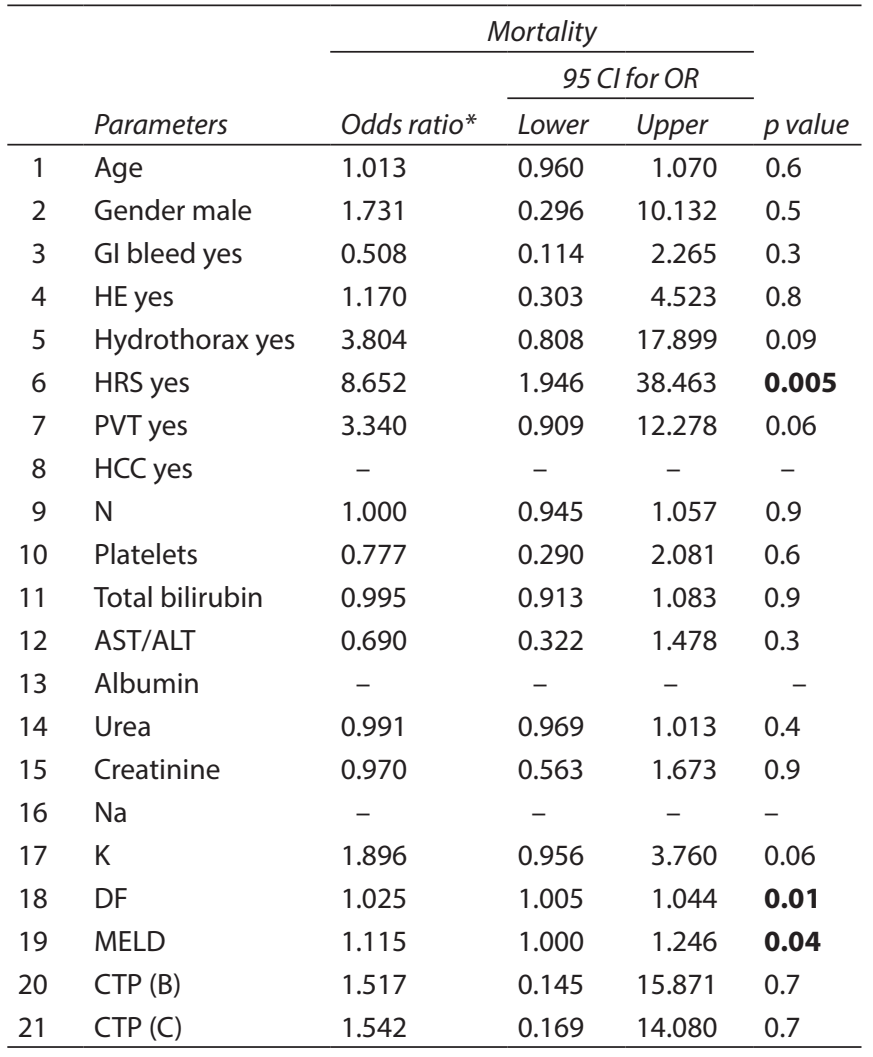

$\mathrm{OR}$, odds ratio; $\mathrm{Cl}$, confidence interval; $\mathrm{HBV}$, hepatitis $\mathrm{B}$ virus; $\mathrm{HCV}$, hepatitis $\mathrm{C}$ virus; $\mathrm{Gl}$, gastrointestinal; $\mathrm{HE}$, hepatic encephalopathy; $\mathrm{HRS}$, hepatorenal syndrome; PVT, portal vein thrombosis; HCC, hepatocellular carcinoma; INR, international normalized ratio; PT, prothrombin time; DF, discriminant function; MELD, model for end-stage liver disease; CTP, Child-TurcottePugh

$p$ values in boldface indicate statistical significance; * obtained using multivariate logistic regression

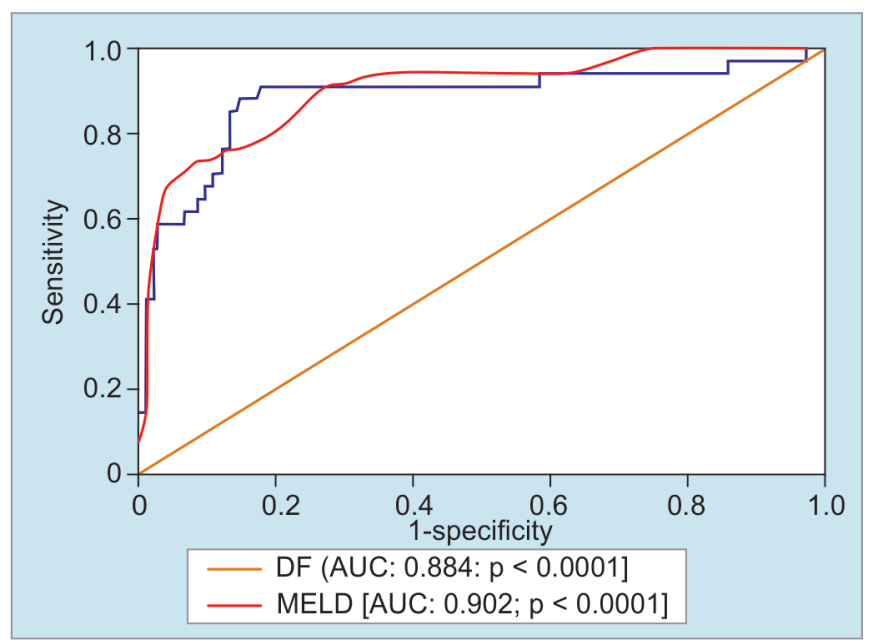

Fig. 2: Receiver-operating characteristic plots for death for parameters Maddrey's discriminant function and model for end-stage liver disease

management of $\mathrm{HE}$ are done rigorously at many centers including at our site. This may be the reason why many of the recent publications have not found $\mathrm{HE}$ as an essential predictor of short-term mortality and morbidity. ${ }^{17,20}$ 
Even though initial assessment had shown significant difference in HE and HCC in patients with cirrhosis, in multivariate analysis the odds were found to be not statistically significant. Other but important complications that have shown to be promising predictors of hospital readmission within 3 months per the current standard of care include hydrothorax, HRS, and PVT. Only HRS carried significant and highest odds of both readmission (24.573) and mortality (8.652). Very few studies have observed HRS as the important predictor of 1-month or 3-month readmission or mortality. ${ }^{11,13,28}$ The important difference can be attributed to the difference in the proportion of patients studied and difference in the etiologies and disease characteristics. Multiple studies have specifically studied these complications with variable results. In the current study, HRS cases contributed $19.39 \%$ (45) of all cases. In one of the studies, in patients with alcoholic liver disease, only HRS was found to be best predictor of short-term mortality. ${ }^{28}$ These findings are according to the established understanding. Hepatorenal syndrome is a frequent complication of end-stage liver cirrhosis. The HRS patients have poor prognosis and their chances of survival are less. Even with established therapies, the profit remains elusive. ${ }^{29}$

Hydrothorax is one of the least encountered complications of cirrhosis but has been a known predictor of readmission. ${ }^{15}$ We observed the predictive nature of hydrothorax only with 3-month hospital readmission with OR of 18.386. No significant odds are observed for mortality. Recent data support the higher significance of hepatic hydrothorax since most of such candidates are considered eligible for orthotopic liver transplantation. ${ }^{30}$

Portal vein thrombosis is generally not reported as important predictors largely because of inadequate sample size of the study population. We observed its impact to be less as compared to hydrothorax and HRS with OR of 5.306. Meta-analysis has predicted the role of PVT in mortality and development of ascites. ${ }^{31}$

Of the scoring systems, DF (OR 1.053), MELD score (OR 1.238), and CTP C (OR 17.276) proved to be important predictors of readmission. But only MELD (OR 1.115) and DF (OR 1.025) showed significant odds for mortality. The CTP C was significant just because it defines a subset of severely decompensated cirrhosis patients vs CTP A or B. One of the most important known factors that predicted 3-month readmission in patients with cirrhosis is MELD score. This study shows the predictive role of the MELD score, which is already used clinically to gauge survival in cirrhotic patients with sepsis, variceal bleeding, fulminate hepatic failure, and alcoholic hepatitis. Incorporating the function of two organs (renal and hepatic), the MELD score has been shown to be a robust predictor of readmission in Indian patients with cirrhosis. ${ }^{11,32}$

Discriminant function, which includes PT and total bilirubin, was also found to be an important predictor of 3-month hospital readmission and mortality. Although commonly used and found effective in prediction of short-term readmission of alcoholic hepatitis, current study suggested its validity in cases of cirrhosis. ${ }^{33}$ Considering ease of use and their origin based on multivariate analysis, the estimated cutoff using ROC to help identify best cases for monitoring purpose to avoid hospital readmission included threshold score of 49.96 for DF and 17.5 for MELD. The threshold for DF was 64.10 and for MELD 26.5 for prediction of 3-month mortality. The threshold was found to be higher as compared to the already published literature. ${ }^{34}$ This can be attributed to improved focused standard of care for advanced cirrhosis. Although the risk factors for 3-month readmission such as HRS, PVT, and hydrothorax are either difficult to modify or nonmodifiable factors, MELD and DF score do help in identification of patients who may benefit from medical intervention. Additional attention if directed toward these patients in the form of careful discharge planning and closer follow-up evaluation and if best be directed toward higher MELD score can definitely benefit patients. In the current study, the independent variables like patient age, gender, and etiology of cirrhosis did not emerge as predictors of 3-month readmission or mortality. Surprisingly the components of Child-Pugh score such as encephalopathy, ascites, bilirubin, albumin, and PT did not predict the short-term readmission or mortality. However, the importance of MELD and DF score and complications like hydrothorax, HRS, and PVT emerged as the most predictive tools and cirrhosis complication to ascertain the rate of readmission and mortality within 3 months of patient discharge.

Strength of the study being that the study results were found to be different from the common consensus, which can be attributed to the recent change in assessment methods and center-specific patient managements. Limitations of this study are many. The results may not be generalized to a wider population but at the same time the patient diversity reported in clinical trials emphasizes centerspecific identification of risk factors and management strategies. Although etiology did not predict readmission in the present study, etiology has a definitive role in the natural history of disease. The present study did not consider the duration of cirrhosis as it could be a definitive risk factor for both readmission and mortality. Adherence to treatment and factors preventing the adherence to treatment among the cirrhotic patients' evaluation would have added more weightage for readmission and mortality of cirrhotic patients.

\section{Conclusion}

The present study revealed that the parameters such as MELD, DF score, and complications such as hydrothorax, HRS, and PVT are the most predictive indicators of cirrhosis complication to ascertain the rate of readmission and mortality within 3 months of patient discharge.

\section{References}

1. Melato M, Sasso F, Zanconati F. Liver cirrhosis and liver cancer. A study of their relationship in 2563 autopsies. Zentralbl Pathol 1993;139(1):25-30.

2. Graudal N, Leth $P$, Marbjerg L, et al. Characteristics of cirrhosis undiagnosed during life: a comparative analysis of 73 undiagnosed cases and 149 diagnosed cases of cirrhosis, detected in 4929 consecutive autopsies. J Intern Med 1991;230(2):165-171. DOI: 10.1111/j.1365-2796.1991.tb00425.x.

3. Lim YS, Kim WR. The global impact of hepatic fibrosis and endstage liver disease. Clin Liver Dis 2008;12(4):733-746. DOI: 10.1016/j. cld.2008.07.007.

4. Murray CJ, Lopez AD. Alternative projections of mortality and disability by cause 1990-2020: global burden of disease study. Lancet 1997;349(9064):1498-1504. DOI: 10.1016/S0140-6736(96)07492-2.

5. Talwalkar JA. Prophylaxis with beta blockers as a performance measure of quality health care in cirrhosis. Gastroenterology 2006;130(3):1005-1007. DOI: 10.1053/j.gastro.2005.11.055.

6. Nguyen GC, Segev DL, Thuluvath PJ. Nationwide increase in hospitalizations and hepatitis $C$ among inpatients with cirrhosis and sequelae of portal hypertension. Clin Gastroenterol Hepatol 2007;5(9):1092-1099. DOI: 10.1016/j.cgh.2007.04.027.

7. Institute of Medicine (U.S.). Crossing the quality chasm: a new health system for the 21st century. Washington, D.C.: National Academy Press; 2001. Committee on Quality of Health Care in America.

8. Phillips CO, Wright SM, Kern DE, et al. Comprehensive discharge planning with post discharge support for older patients with 
congestive heart failure: a meta-analysis. JAMA 2004;291(11):13581367. DOI: 10.1001/jama.291.11.1358.

9. Hunt SA, Abraham WT, Chin MH, et al. ACC/AHA 2005 guideline update for the diagnosis and management of chronic heart failure in the adult: a report of the American College of Cardiology/American Heart Association Task Force on Practice Guidelines (Writing Committee to Update the 2001 guidelines for the evaluation and management of heart failure): developed in collaboration with the American College of Chest Physicians and the International Society for Heart and Lung Transplantation: endorsed by the Heart Rhythm Society. Circulation 2005;112(12):e154-e235. DOI: 10.1161/CIRCULATIONAHA.105.167586.

10. Volk ML, Tocco RS, Bazick J, et al. Hospital re-admissions among patients with decompensated cirrhosis. Am J Gastroenterol 2012;107(2):247-252. DOI: 10.1038/ajg.2011.314.

11. Berman K, Tandra S, Forssell K, et al. Incidence and predictors of 30-day readmission among patients hospitalized for advanced liver disease. Clin Gastroenterol Hepatol 2011;9(3):254-259. DOI: 10.1016/ j.cgh.2010.10.035.

12. Ganesh S, Rogal SS, Yadav D, et al. Risk factors for frequent readmissions and barriers to transplantation in patients with cirrhosis. PLoS One 2013;8(1):e55140. DOI: 10.1371/journal.pone.0055140.

13. Seraj SM, Campbell EJ, Argyropoulos SK, et al. Hospital readmissions in decompensated cirrhotics: factors pointing toward a prevention strategy. World J Gastroenterol 2017;23(37):6868-6876. DOI: 10.3748/ wjg.v23.i37.6868.

14. Tapper EB, Halbert B, Mellinger J. Rates of and reasons for hospital readmissions in patients with cirrhosis: a multistate population-based cohort study. Clin Gastroenterol Hepatol 2016;14(8):1181-1188. DOI: 10.1016/j.cgh.2016.04.009.

15. Bajaj JS, Reddy KR, Tandon P, et al. The 3-month readmission rate remains unacceptably high in a large North American cohort of patients with cirrhosis. Hepatology 2016;64(1):200-208. DOI: 10.1002/ hep.28414.

16. Durand F, Valla D. Assessment of the prognosis of cirrhosis: Child-Pugh versus MELD. J Hepatol 2005;42(Suppl 1):S100-S107. DOI: 10.1016/ j.jhep.2004.11.015.

17. Patel R, Lotlikar MS. Predictors of 1-month and 3-months hospital readmissions in decompensated cirrhosis in India. J Clin Exp Hepatol 2017;7(1):S53. DOI: 10.1016/j.jceh.2017.05.100.

18. Daswani R, Kumar A, Anikhindi SA, et al. Predictors of 90-day mortality in patients with severe alcoholic hepatitis: experience with 183 patients at a tertiary care center from India. Indian J Gastroenterol 2018;37(2):141-152. DOI: 10.1007/s12664-018-0842-0.

19. Kartoun U, Corey KE, Simon TG, et al. The MELD-plus: a generalizable prediction risk score in cirrhosis. PLoS One 2017;12(10):e0186301. DOI: 10.1371/journal.pone.0186301.

20. Tapper EB, Finkelstein D, Mittleman MA, et al. Standard assessments of frailty are validated predictors of mortality in hospitalized patients with cirrhosis. Hepatology 2015;62(2):584-590. DOI: 10.1002/ hep. 27830 .

21. Sharma B, Raina S, Marwah R, et al. A follow-up study on adult patients with cirrhosis recruited in an open cohort from the hills of Himachal Pradesh. Int J Health Allied Sci 2016;5:24-27. DOI: 10.4103/2278344X.173879.

22. Bhattacharyya M, Barman NN, Goswami BB. Clinical profile of cirrhosis of liver in a tertiary care hospital of Assam, North East India. IOSR J Dent Med Sci 2016;15(1):21-27.

23. Sajja KC, Mohan DP, Rockey DC. Age and ethnicity in cirrhosis. J Investig Med 2014;62(7):920-926. DOI: 10.1097/JIM.0000000000000106.

24. Chen CY, Wu CJ, Pan CF, et al. Influence of age on critically III patients with cirrhosis. Int J Gerontol 2015;9:233-238. DOI: 10.1016/ j.ijge.2014.10.003.

25. Angele MK, Pratschke S, Chaudry IH. Does gender influence outcomes in critically ill patients? Crit Care 2012;16(3):129. DOI: 10.1186/CC11365.

26. Kalaitzakis E, Josefsson A, Björnsson E. Type and etiology of liver cirrhosis are not related to the presence of hepatic encephalopathy or health-related quality of life: a cross-sectional study. BMC Gastroenterol 2008;8:46. DOI: 10.1186/1471-230X-8-46.

27. Saffouri E, Lim ES, Kim SW, et al. Short-term changes in liver tests predict long-term mortality. Frontline Gastroenterol 2016;7(4): 234-239. DOI: 10.1136/flgastro-2015-100669.

28. Potts JR, Goubet S, Heneghan MA, et al. Determinants of long-term outcome in severe alcoholic hepatitis. Aliment Pharmacol Ther 2013;38(6):584-595. DOI: 10.1111/apt.12427.

29. Appenrodt B, Zielinski J, Brensing KA, et al. Degree of hepatic dysfunction and improvement of renal function predict survival in patients with HRS type I: a retrospective analysis. Eur J Gastroenterol Hepatol 2009;21(12):1428-1432. DOI: 10.1097/MEG.0b013e32832ec16a.

30. Garbuzenko DV, Arefyev NO. Hepatic hydrothorax: an update and review of the literature. World J Hepatol 2017;9(31):1197-1204. DOI: 10.4254/wjh.v9.i31.1197.

31. Stine JG, Shah PM, Cornella SL, et al. Portal vein thrombosis, mortality and hepatic decompensation in patients with cirrhosis: a meta-analysis. World J Hepatol 2015;7(27):2774-2780. DOI: 10.4254/wjh.v7.i27.2774.

32. Peng Y, Qi X, Guo X. Child-Pugh versus MELD score for the assessment of prognosis in liver cirrhosis: a systematic review and meta-analysis of observational studies. Medicine (Baltimore) 2016;95(8):e2877. DOI: 10.1097/MD.0000000000002877.

33. Sheth M, Riggs M, Patel T. Utility of the mayo end-stage liver disease (MELD) score in assessing prognosis of patients with alcoholic hepatitis. BMC Gastroenterol 2002;2:2. DOI: 10.1186/1471-230X-2-2.

34. Luca A, Miraglia R, Maruzzelli L, et al. Early liver failure after transjugular intrahepatic portosystemic shunt in patients with cirrhosis with model for end-stage liver disease score of 12 or less: incidence, outcome, and prognostic factors. Radiology 2016;280(2):622-629. DOI: 10.1148/radiol.2016151625. 